\title{
Isolation and Characterization of an Algicidal Bacterium in biofilm on submerged reed stems for control of Microcystis blooms
}

\author{
Yan Rongjun \\ College of Ocean, Zhejiang University of Technolog. Hangzhou, China. \\ Email: yanrj@zjut.edu.cn
}

\begin{abstract}
Keywords: reed; biofilm, Algae lysis bacteria; algae lysis mechanism; 16S rDNA; Bacillus
Abstract. From the reed surficial biofilm isolated a strain of algicidal bacteria B2 have significant algicidal effect of Microcystis aeruginosa. By means of microscope and scanning electron microscope and monitoring the change of $\mathrm{pH}$ in the medium of $\mathrm{B} 2$, we found that the dissolved algae bacteria B2 was dissolved in algae. Algicidal way for bacteria secrete soluble active substances Algicidal algae. Analysis of $16 \mathrm{~S}$ rDNA sequence revealed that strain B2 belonged to the genus Brevundimonas. By 16S rRNA sequence analysis and data in GenBank homology search, combined with morphological, physiological and biochemical characteristics of the bacterial strains belonging to the genus Bacillus.
\end{abstract}

\section{Introduction.}

Water eutrophication of water bodies leading to overgrowth of algae, especially cyanobacteria outbreak is caused by the formation of algae blooms. After the death of a large number of algae in its decomposition process, not only the stench, destroy the landscape, while a large consumption of dissolved oxygen in the water, so fish suffocation. More importantly, the algae can release biological toxins - microcystins. Aquatic life, drinking water safety and human health poses a great threat.

This paper reports the reed as an object, separating one pair screened Microcystis aeruginosa significantly Algicidal role and ability to stabilize the Algicidal Algicidal Bacillus aid of a microscope and scanning electron microscopy analysis of the strain globosa algicidal effects and lytic character, and by $16 \mathrm{~S}$ rDNA sequencing analysis to explore the bacterial community composition. The results contribute to a better understanding of the interaction between bacteria and algae processes and mechanisms in order to provide practical guidance and possible ways to curb blooms microbial control.

\section{Materials and methods}

\section{Cyanobacterial cultures}

Microcystis aeruginosa was purchased from the Freshwater Algae Culture Collection of the Institute of Hydrobiology, Chinese Academy of Sciences.from Wuhan aquaculture. The M.aeruginosa strains were maintained as unialgal axenic cultures at $28^{\circ} \mathrm{C}$, with illumination at 150 $\mu \mathrm{mol}$ photons $\mathrm{m}^{-2} \mathrm{~s}^{-1}$ under a $12-\mathrm{h}$ light/dark regimen. The cyanobacterial cells were incubated in BG11 medium (100 $\mathrm{mL})$ adjusted to $\mathrm{pH} 7.0$.

\section{Algicidal bacteria isolated, purified and screened}

The natural growth of reeds was collected, frozen back to the lab; in the audience stripped clean work surface biofilm reeds, isolated biofilm suspension. After coarse filtration membrane $1 \mu \mathrm{m}$, with sterile water and the filtrate was diluted 1000 fold gradient . Pour plate method using the bacterial inoculum was diluted 1000 fold from the suspension to a biofilm on a solid medium, cultured under $25{ }^{\circ} \mathrm{C} 72 \mathrm{~h}$, characterized by having distinct bacterial colonies picked from the plate and from specific screening, taking partition scribing method, the strain was further purified.

\section{Isolation and Identification of Algae-lysing Bacteria}

The bacterium was identified algicidal bacteria after the bacteria species were identified. In this experiment, bacterial universal primers $27 \mathrm{~F}$ and 1492R, through the bacterial 16S rDNA sequence 
homology. PCR products AC Beijing Liuhe Genomics Technology Co. AG sequenced.The sequencing results were compared enter the NCBI data base, search homologous DNA sequences and compared . Applications clustalx and MEGA5 biological software phylogenetic tree was constructed using the Neighbor-Joining method.

\section{Results}

\section{Isolation and identification of algicidal bacteria}

The morphology of algal cells in different sampling time was shown in the microscope by the microscope. Figure 1 (a) is the normal cells of Microcystis aeruginosa; in 1D in the algal culture medium adding B2 on Microcystis aeruginosa growth had no obvious effect, also observed under the microscope, adding algicidal bacteria B2 shape of algae cells see [figure 1 (b)), no significant changes. With the addition of bacterial B2 and the activity of algae, the algae cells were destroyed in a large amount, from Figure 1 (c), the algae cell debris could be clearly seen, and the color of the algae cells changed to shallow and scattered. Until the end of culture, that is, the first $14 \mathrm{~d}$, the majority of the culture fluid is broken algal cell debris [figure 1 (d)], the culture solution failed to see the complete algae cells, algae culture into turbidity.

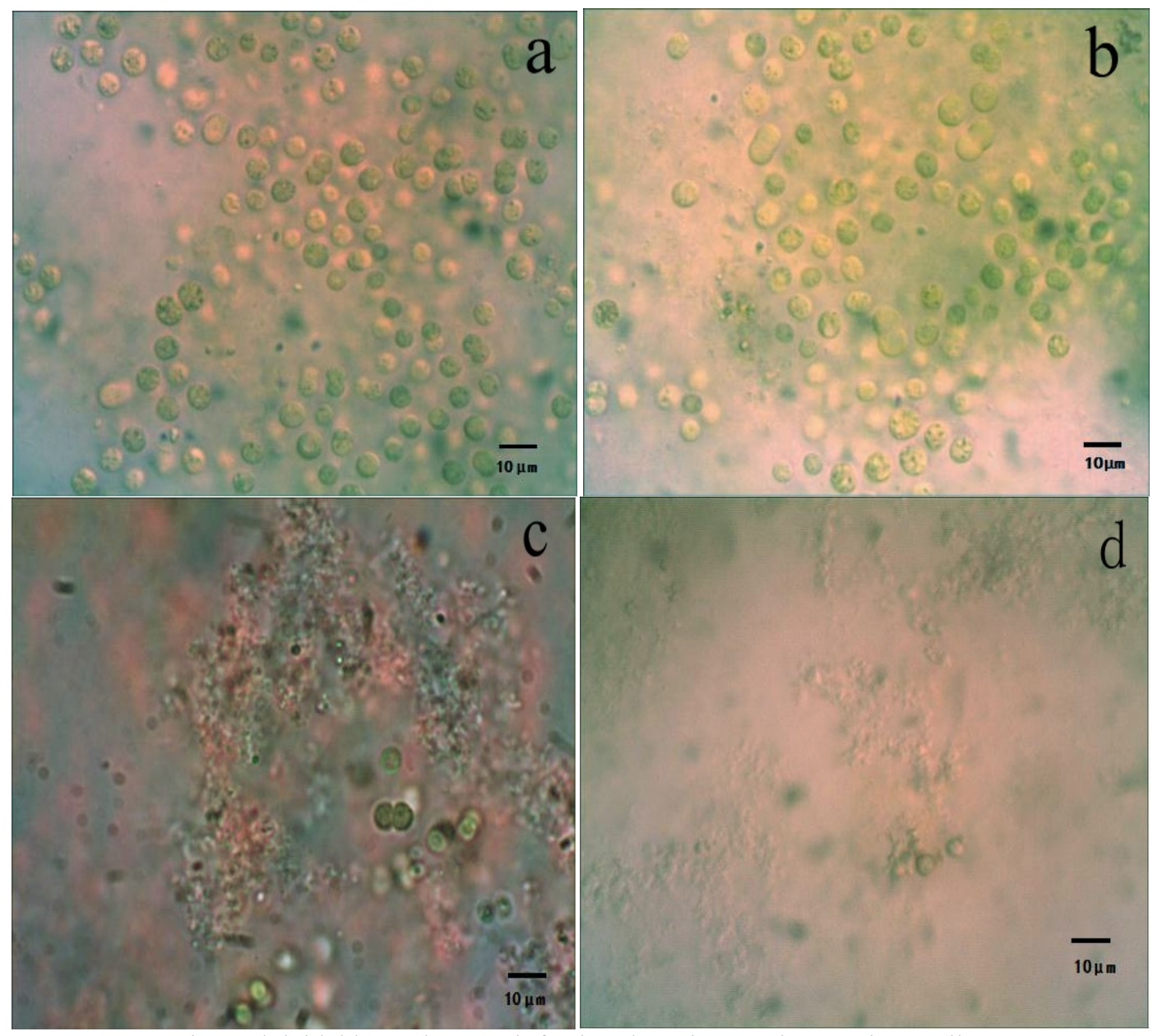

Fig.1 Algicidal bacterium B2 infecting the Microcystis aeruginos cells

\section{Determination of algicidal activity}

Scanning electron microscopy was used to observe the morphological changes of algae cells. From Figure 2, we can see that the bacterial B2 has the function of dissolving (a, To cell shape is circular in shape.; b. figure algal cell disruption, cell morphology defect, did not find direct contact with bacteria and algae cells) Soluble algae bacterium B2 of Microcystis aeruginosa process: 1, Microcystis aeruginosa by bacterial stress accumulation; 2, release of algicidal substances algae lysing algae bacteria soluble; 3 , part of Microcystis aeruginosa surface cracking; 4, in the algal cells dissolved 
substances, Microcystis aeruginosa is dissolved. Therefore, it should belong to the above second ways, that is, the indirect lysis of B2 cells.
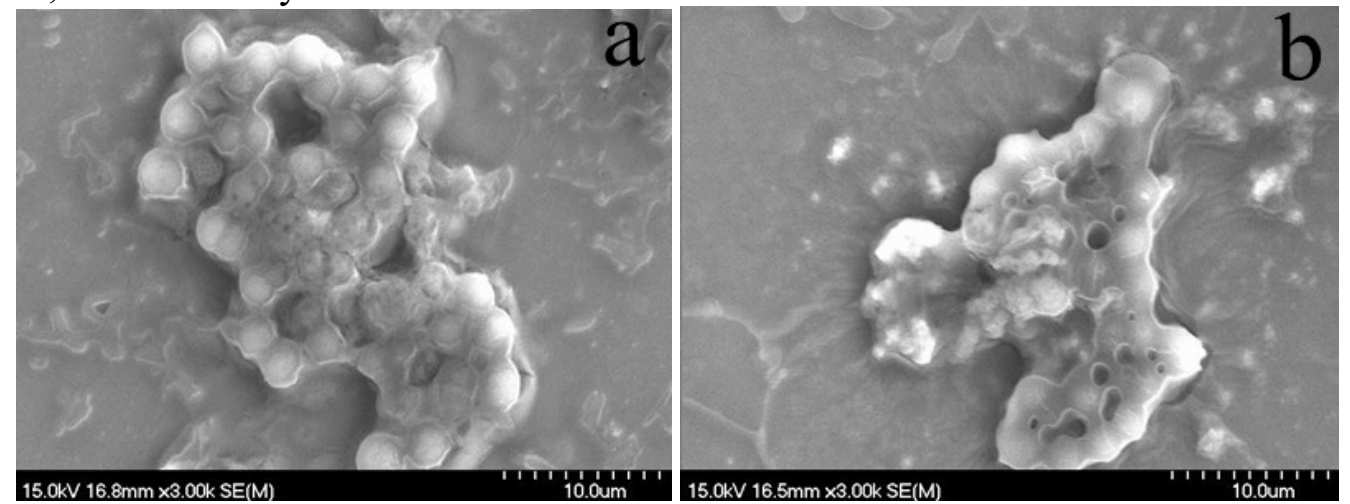

Fig.2SEM views of Algicidal bacteria B2 infecting the Microcystis aeruginosa cells (Scale bars $=10 \mu \mathrm{m})$

\section{Isolation and Identificaon of Algae-lysing Bacteria}

Algicidal bacteria to improve the separation efficiency and strengthen research in molecular biology Algicidal algicidal bacteria is one of the elements of bacterial research . Currently 16S rDNA sequence similarity analysis is a key indicator given genus of bacteria B2 bacterial 16S rDNA gene after approximately $1.5 \mathrm{~Kb}$ PCR amplified fragment ( Figure 5 ). On B2 of 16S rDNA gene sequencing to obtain the length of $16 \mathrm{~S}$ rDNA sequences $1398 \mathrm{bp}$. After submitting to the GenBank database using BLAST alignment analysis, phylogenetic tree ( Figure 3 ), the strain of Bacillus thuringiensis with the closest relationship , up to $97 \%$ similarity. Algicidal bacteria belonging to the genus Bacillus.

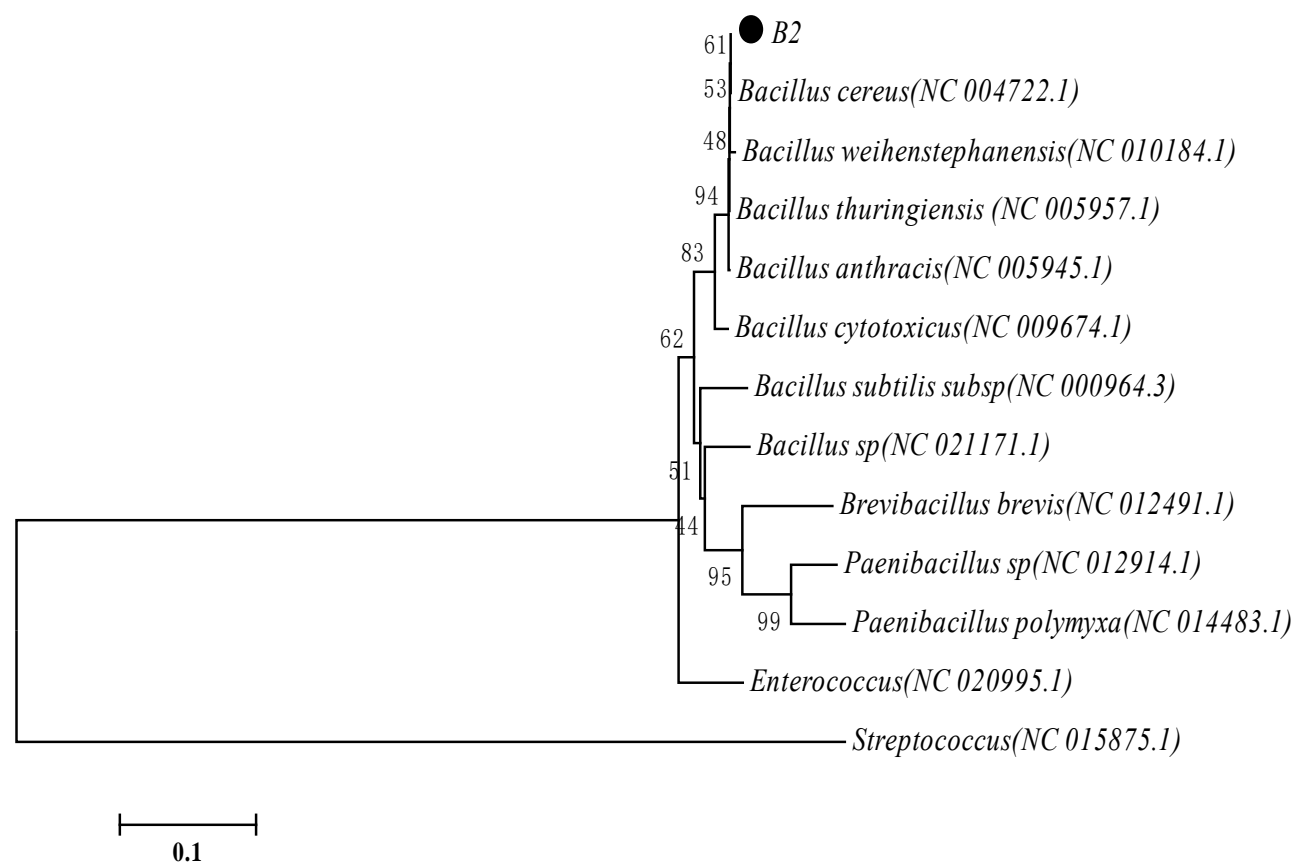

Figure 3. Phylogene tree based on bacterial $16 \mathrm{~S}$ rDNA gene sequence of the isolate $\mathrm{B} 2$ strain and closely related members. Numbers at nodes are levels of bootstrap support (\%). The codes after the names are Genebank Accession numbers

\section{Conclusions}

The results show that not only one-sided algicidal effect but also two-sided reciprocal inhibition interactions exist between algicidal bacteria and cyanobacteria, indicating the complexity of cyanobacteria-algicidal bacteria interactions responses into consideration when assessing potential use of algicidal bacteria. 


\section{Acknowledgements}

This work was financially supported by the Zhejiang Natural Science Foundation (LY14E090008).

\section{References}

Li L, Xie P. Hepatic histopathological characteristics and antioxidant response of phytoplanktivorous silver carp intraperitoneally injected with extracted microcystins. Biomed

Environ Sci, 2009; 22, 297 - 302.7.

Ma, R., Kong, F., Duan, H., Zhang, S., Kong, W., Hao, J., 2008. Spatio-temporal distribution of cyanobacteria blooms based on satellite imageries in Lake Taihu, China (in Chinese, with English abstract). J. Lake Sci. 20, 687-694.

Mayali, X., Azam, F., 2004. Algicidal bacteria in the sea and their impact on algal blooms. J. Eukaryot. Microbiol. 51, 139-144.

Wang, X., Gong, L., Liang, S., Han, X., Zhu, C., Li, Y., 2005. Algicidal activity of rhamnolipid biosurfactants produced by Pseudomonas aeruginosa. Harmful Algae 4 (2), 433-443.

Su, J.Q., Yang, X.R., Zheng, T.L., Tian, Y., Jiao, N.Z., Cai, L.Z., Hong, H.S., 2007. Isolation and characterization of a marine algicidal bacterium against the toxic dinofla-gellate Alexandrium tamarense. Harmful Algae 6 (6), 799-810. 